\title{
The probabilistic approach to human reasoning
}

\section{Mike Oaksford and Nick Chater}

\begin{abstract}
A recent development in the cognitive science of reasoning has been the emergence of a probabilistic approach to the behaviour observed on ostensibly logical tasks. According to this approach the errors and biases documented on these tasks occur because people import their everyday uncertain reasoning strategies into the laboratory. Consequently participants' apparently irrational behaviour is the result of comparing it with an inappropriate logical standard. In this article, we contrast the probabilistic approach with other approaches to explaining rationality, and then show how it has been applied to three main areas of logical reasoning: conditional inference, Wason's selection task and syllogistic reasoning.
\end{abstract}

In a standard reasoning task, performance is compared with the inferences peopleshould make according to logic, so a judgement can bemade on the rational ity of people's reasoning. It has been found that peoplemakelargeand systematic (i.e. nonrandom) errors ${ }^{1,2}$, which suggests that humans might be irrational ${ }^{3,4}$. However, the probabilistic approach argues against this interpretation. Rather than view this behaviour as error proneit is argued that performance has been compared with the wrong normativestandard. When the comparison is with probability theory rather than logic, participants' reasoning is seen in a more positivelight. This approach was first applied to Wason's selection task ${ }^{5}$, wheremany model s have been proposed ${ }^{6-11}$ and investigated ${ }^{12-17}$. More recently the probabilistic approach has been extended to the other coreareas of thepsychology of reasoning: syllogisms ${ }^{18-20}$ and conditional inference ${ }^{21-24}$, wheretheneed for a probabilistic approach has been most apparent ${ }^{25-29}$.

We first contrast the probabilistic approach with other approaches to human rationality. Wethen discuss theinadequacy of logic as an account of everyday reasoning, and show why a probabilistic approach looks more promising. Wethen review its application to explaining the coretasks in reasoning research (seeabove).

\section{Rationality and theories of reasoning}

Both mental-logic approache ${ }^{30}$ and mental-model

Mike Oaksford*

School of Psychology,

Cardiff University, PO Box

901, Cardiff, UK CF1 3YG.

*e-mail:

oaksford@cardiff.ac.uk

Nick Chater

Dept of Psychology,

University of Warwick

Coventry, UK CV2 7AL. are rational in principlebut err in practice; that is, people's logical reasoning algorithms aresound but constrained by cognitivelimitations.

Theseapproaches arehard to reconcilewith two facts. First, error rates can beas high as $96 \%$ (in Wason's selection task). Second, everyday rationality in guiding thought and action seems to behighly successful. How can this success be understood if peoples' reasoning system is proneto so much error?

Other theorists distinguish twotypes of rationality to resolvethis apparent conflict ${ }^{32}$. Everyday rationality does not depend on formal systems like logic and only formal rational ity is constrained and error prone. But how then is thesuccess of everyday inference to be explained? Therewould appear to be no obvious al ternative, asidefrom arguing that everyday rationality is also based on formal principles of reasoning. But this seems to bring us full circle.

The probabilistic approach resolves this problem. Everyday reasoning is probabilistic and peoplemake errors in so-cal led logical tasks because they generalizethesestrategies tothelaboratory. This approach has been much influenced by Anderson's account of rational analysi s $^{32-36}$. Other authors have also questioned theappropriateness of thenormative theories used to assess human reasoning 37,38 . According to the probabilisticapproach much experimental research in the'psychology of deductive reasoning' does not engage people in deductive reasoning at all but rather engages strategies suitable for everyday reasoning. In thenext section weexplain why everyday reasoning is best characterized probabilistically rather than logically.

\section{Everyday inference: logic or probability?}

Everyday inferences are uncertain. Supposesomeone believes that birds fly, it is not clear how to capture the patterns of inferencethat this everyday generalization permits. For example, standard logic, which mental logicand mental models assume to be normative, will not do because it allows 'strengthening of theantecedent'. Formalizing this generalization as 'if something is a bird it flies', entails that if someoneknows that Tweety is a bird, they can infer that Tweety flies. However, strengthening of the antecedent means that when given further information, likeTweety is an Ostrich, they should still infer that Tweety flies. But, intuitively, this new piece of information should defeat the previous conclusion: everyday inferences are defeasible. There 


\section{Box 1 Logic and everyday reasoning}

Standard logic cannot account for conditionals in everyday inference. An alternative account of conditionals is given by the Lewis-Stalnaker possible-worlds semantics ${ }^{a, b}$. The intuitive idea for a counterfactual conditional such as 'if Tweety had been a bird, he would have been able to fly' is that in the world maximally similar to the actual world $(\alpha)$ but in which Tweety was a bird, he could fly. This semantics can be applied to the indicative conditional (where we simply don't know whether Tweety is a bird). When this is done it is clear that strengthening of the antecedent (see main text) cannot hold. For example, 'if it's a bird, then it flies' does not imply that 'if it's a bird and it's an ostrich, then it flies'. The worlds in which the antecedents are evaluated will differ - the world most similar to $\alpha$ in which something is a bird is not the same as the world most similar to $\alpha$ in which something is an ostrich. In particular, in the first world, the thing will most probably fly (because most birds fly); but in the second world, the thing will not fly (because ostriches can't fly). However, for psychological purposes we need an account of the formal processes that can implement this semantics. The programme of attempting to mechanize reasoning about the way the world might be, has been taken up by the study of knowledge representation in artificial intelligence (AI). However, it is far from clear that formal attempts in Al can capture the Lewis-Stalnaker semanticsc.

Problems arise when the inferences that can be made from one antecedent intuitively conflict with the inferences that can be made from another. For example, knowing that 'Tweety is a sparrow' leads to the conclusion that 'Tweety flies', whereas knowing that 'Tweety is one second old' leads to the conclusion that 'Tweety cannot fly'. This leads to the problem of what we infer when we learn that 'Tweety is a one-second-old sparrow', that is, when the antecedent is strengthened. It is intuitively obvious that a one-second-old sparrow cannot fly. However, formally, it is not obvious how to capture this conclusion. We can formally regard these two pieces of information as two conditional rules: if something is a bird it can fly, and if something is one second old it cannot fly. Formal proposals in Al appear to be unable to break the symmetry between these rules and specify which of these conflicting conclusions we should accept ${ }^{d}$. There have been various alternative proposals in Al that attempt to deal with this problem of strengthening the antecedent, or default reasoning ${ }^{\mathrm{d}-\mathrm{g}}$. However, these approaches ${ }^{\text {h-j }}$ all seem to fall foul of similar problems. Moreover, mental logics ${ }^{k}$ and mental models' also fail to address these issues because they formalize the conditional using standard logic. However, as we have seen, standard logic is unable to capture the use of conditionals in everyday inference.

\footnotetext{
References

a Lewis, D. (1973) Counterfactuals, Oxford University Press

b Stalnaker, R. (1968) A theory of conditionals. In Studies in Logical Theory (Rescher, N., ed.), pp. 98-112, Oxford University Press.

c Pylyshyn, Z.W. and Ford, K.M. (1995) TheRobot's Dilemma Revisited, Ablex Publishing

d Reiter, R. (1985) On reasoning by default. In Readings in Knowl edgeRepresentation.

(Brachman, R. and Levesque, H., eds), pp. 401-410, Morgan Kaufmann

e McCarthy, J .M. (1980) Circumscription: a form of non-monotonic reasoning. Artif. Intell. 13, 27-39

f McDermott, D. and Doyle, J . (1980) Non-monotonic logicl. Artif. Intell. 13, 41-72

g McDermott, D. (1982) Non-monotonic logic: II . Non-monotonicmodal theories. J . Assoc. Comput. Mach. 29, 33-57

h McDermott, D. (1987)A critique of pure reason. Comput. Intell. 3, 151-160

i Shoam, Y. (1987) Reasoning About Change, MIT Press

j Shoam, Y. (1988) Efficient reasoning about rich temporal domains. J . Philos. Logic $17,443-474$

k Rips, L.J . (1994) ThePsychology of Proof, MIT Press

I J ohnson-Laird, P.N. and Byrne, R.M.J . (1991) Deduction, Erlbaum
}

are a number of logical proposals to get around this problem (seeBox 1) but none appears to succeed ${ }^{39-44}$.

The probabilistic approach handles this case naturally by interpreting conditionals using conditional probability. Thus, thestatement birdsfly claims that the conditional probability of something flying, given that it is a bird, is high. Probability theory naturally captures defeasibility. I f all that is known is that something is a bird, then the probability that it flies might be, say, $0.9[\mathrm{P}$ (flies| bird) $=0.9]$. However, the probability of it flying given that it is a bird and an ostrich is near $0[\mathrm{P}(\mathrm{flies} \mid$ bird, ostrich $) \approx 0$ ], and the probability of it flying given that it is a bird and a parrot may be, say $0.96[P(f l i e s \mid$ bird, parrot $)=0.96]$. All thesestatements are compatibleaccording to probability theory. Sotheresult of strengthening the antecedent leads to acceptable results. This approach tothemeaning of conditional statements was proposed in phi losophy by Adams $\mathrm{s}^{45,46}$, and has been used in artificial intelligenceby Pearl 47,48 .

\section{Everyday inference in the laboratory}

Wenow review in detail how the probabilistic approach has been applied in thethree main areas of human reasoning research. We present them in theorder of inferential complexity (least first). We concentrate on our own research because themodels wehaveproposed all explicitly aim toshow how biases in human reasoning can havea rational basis. Thus, they attempt to resolvethe paradox of why such a successful organism should appear soirrational in thelaboratory.

\section{Conditional inference}

Conditional inferenceinvolves presenting participants with a conditional premise, if $p$ then $q$, and one of four categorical premises, $p$, not-p, $q$, or not-q. Logically, given $p$ participants should draw the conclusion q and given not-q they should draw the conclusion not-p. Thesearethelogically val id inferences of modus ponens (MP) and modus tollens (MT) respectively. Moreover, given not-p participants should not draw the conclusion not-q and given q they should not draw the conclusion $p$. These arethe logical fallacies of denying theantecedent (DA) and affirming the consequent (AC) respectively. So, logically, participants should endorse MP and MT equally and they should refuseto endorse DA or AC. However, in fact they endorse MP significantly more than MT and they endorseDA and AC at levels significantly abovezero.

Following other researchers in this area ${ }^{25-29}$ we proposed a model of conditional reasoning based on conditional probability ${ }^{21}$. Thegreater the conditional probability of an inferencethemoreit should be endorsed. The meaning of a conditional statement was defined in a two by two contingency table ${ }^{7,21}$ (Fig. 1).

The contingency table represents a conditional rule, if $p$ then $q$, wherethere is a dependency between the $p$ and q that may admit exceptions $(\varepsilon)$. a is the probability of theantecedent, $P(p)$. b is the probability of the 
Fig. 1. The contingency table for a conditional rule, if $p$ then $q$, where there is a dependency between the $p$ and $q$ that may admit exceptions $(\varepsilon)$. $a=P(p), b=P(q)$, and $\varepsilon=P($ not $-q \mid p)$

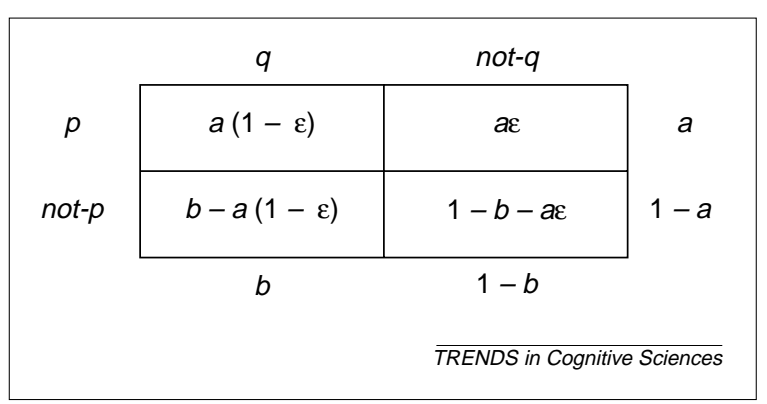

consequent, $\mathrm{P}(\mathrm{q}) . \varepsilon$ is the probability of exceptions; that is, theprobability that $q$ does not occur even though $p$ has, $\mathrm{P}($ not-q| $\mathrm{p})$. Conditional probabilities for each inference werethen derived. For example, the conditional probability for MP, that is, $P(q \mid p)=1-\varepsilon$, depends only on the probability of exceptions. With few exceptions the probability of drawing the MP inference will behigh. However, the conditional probability of MT [that is, $P($ not-p| not- $q)=(1-b-a \varepsilon) /(1-b)]$ depends on the probability of theantecedent, $P(p)$, and the probability of the consequent, $P(q)$, as well as the probability of exceptions. As longas thereare exceptions $(\varepsilon>0)$ and the probability of theantecedent is greater than the probability of the consequent not occurring $[P(p)>1-P(q)]$, then the probability of $M T$ is less than MP $[P($ not-p| not-q) $<P(q \mid p)]$. For example, if $P(p)=0.5, P(q)=0.8$ and $\varepsilon=0.1$, then $P(q \mid p)=0.9$ and $P($ not-p $\mid$ not-q $)=0.75$.

This model accounts for the people's preferencefor MP over MT. The conditional probabilities associated with DA and AC also depend on these parameters, which means that they can benon-zero. Consequently the model al so predicts that the fallacies should be endorsed to some degree. This model has also been applied to a variety of other effects in conditional inference ${ }^{21,22}$ (see Box 2).

In the conditional probability model, and in others ${ }^{25,27,28}$, theconditional premisealoneis regarded as uncertain. But what happens when the categorical premise is also uncertain? Peopletend to endorse conclusions that are only as probableas theleast probable premise $e^{26,29}$. This is consistent with probability theory becausethe probability of the conclusion can beregarded as the product of the probabilities of thetwo premises ${ }^{26,29}$. For example, for $D A$, the probability that the conclusion not-q is endorsed is $P$ (not-q| not-p).P(not-p). Of course, when the categorical premise, not-p, is certain $[P($ not- $p)=1]$, then this account reduces to the model above.

However, somerecent research ${ }^{26}$ has shown behaviour less consistent with probability theory and more consistent with al ternativeformalisms for dealing with uncertainty, such as Dempster-Shafer theory ${ }^{49}$.

Wason's selection task

The probabilistic approach was originally applied to Wason's selection task, in which participants must select cards to find out whether a rule [e.g. 'if there is an $A$ on oneside of thecard ( $p$ ) there is a 2 on the other side (q)'] is true or false ${ }^{6,50,51}$. Participants see four cards, onewith A showing (p), onewith K (not-p), onewith 2 (q) and onewith 7 (not-q). They are told to select only those they must turn in order to find out whether theruleis true or false.

In theinformation-gain model ${ }^{6}$ peopleare assumed to select evidence (i.e. turn cards) to determine whether q depends on $p$, as in Fig. 1 (the dependence hypothesis, $H_{D}$ ), or whether $p$ and $q$ are statistically independent (the independence hypothesis, $\mathrm{H}_{1}$ ). Participants are looking for evidence that provides themost discrimination between these two hypotheses. Initially, participants are maximally uncertain about which is true; that is, the prior probabilities of $\mathrm{H}_{\mathrm{D}}$ and $\mathrm{H}_{1}$ are each 0.5 .

The participants' goal is to select evidence (turn cards) that would be expected to producethegreatest reduction in this uncertainty. This involves calculating the posterior probabilities that the hypotheses, $\mathrm{H}_{\mathrm{D}}$ or $\mathrm{H}_{1}$, aretrue given someevidence. These probabilities are calculated using Bayes' theorem, which requires information about prior probabilities $\left[P\left(H_{D}\right)=P\left(H_{1}\right)=0.5\right]$ and the likelihoods of evidence given a hypothesis; for example, the probability of finding an A when turning the 2 card, assuming $\mathrm{H}_{D}\left[P\left(A \mid 2, H_{D}\right)\right]$. Theselikelihoods can be calculated directly from the contingency tables for each hypothesis: for $\mathrm{H}_{\mathrm{D}}$, the contingency tablein Fig. 1, and for $\mathrm{H}_{1}$, theindependence model, wherethe cell values are simply the products of the marginals. The expected reduction in uncertainty by turning any of thefour cards can then becalculated.

Assuming that themarginal probabilities $P(p)$ and $P(q)$ are small (the 'rarity assumption'), the $p$ and the q cards would beexpected to provide the greatest reduction in uncertainty about which hypothesis was true. Consequently, theselection of cards that seemingly demonstrates human irrational ity might reflect a rational data-sel ection strategy. I ndeed this strategy might be optimal in an environment where most properties arerare- for example, most things are not black, not ravens, and not apples - but this has been disputed 9,50 .

This model can account for most of thefindings on the selection task (see Box 3) and it has been defended2,51 against a variety of objections $5^{9,10,17,52-56}$. Therehas also been much research testing the empirical predictions of this and alternative probabilistic models $\mathrm{s}^{10,11,13-16}$. Alternativemodels ${ }^{50}$ takeeither a 'disinterested' approach to human inquiry ${ }^{6,11}$ or a 'decision-theor etic' approach 9,10 . The disinterested approach was outlined above. Disinterested approaches, liketheinformation-gain model, makeno assumptions about the val ue people might or might not place on particular types of evidence. By contrast, decision-theoretic approaches expl icitly introducetheseutilities intothe decision to select a card. They makethe same predictions for most of the data but diverge when people do not believea hypothesis. On the decision-theoretic view, 


\section{Box 2. Explaining conditional inference}

The behaviour of the conditional-inference model is shown in Fig. I. This behaviour explains the two principal effects observed in conditional inference: negative conclusion bias, and suppression effects. Negative conclusion bias arises in Evans' negation paradigm $^{\mathrm{a}-\mathrm{c}}$. Here, negations are used in the antecedents and consequents of the rules to createfour taskrules (where $\mathrm{A}=$ Affirmative, $\mathrm{N}=$ Negative): if $p$ then $q(\mathrm{AA})$; if $p$ then not- $q$ (AN); if not- $p$ then $q$ (NA); and if not- $p$ then not- $q$ (NN). This manipulation means that half the conclusions of any inference will be affirmative and half of them will be negative. 'Negative conclusion bias' is observed when participants endorse more inferences with a negative conclusion than with an affirmative conclusion. Negated categories have a higher probability than their affirmative counterparts; for example, $P(x$ is a dog $)<P(x$ is not a dog)d,e. Consequently if a conclusion is negated then it corresponds to a high-probability conclusion. The probability of the conclusion is on the $x$-axis in Fig. I. It is clear that as this probability increases so the probability with which the model predicts an inference should beendorsed also increases. So the model can explain negative conclusion bias. Recentexperiments are broadly consistent with this account ${ }^{f-h}$.

Suppression effects occur when further information reduces the degree to which an inference is endorsed. For example, if someone is told that 'if the key is turned the car starts' and that 'the key is turned', they are likely to infer that 'the car starts', by modus ponens (MP). However, if they are also told that ' if the petrol tank is not empty the car starts', they are less likely to endorse (a)

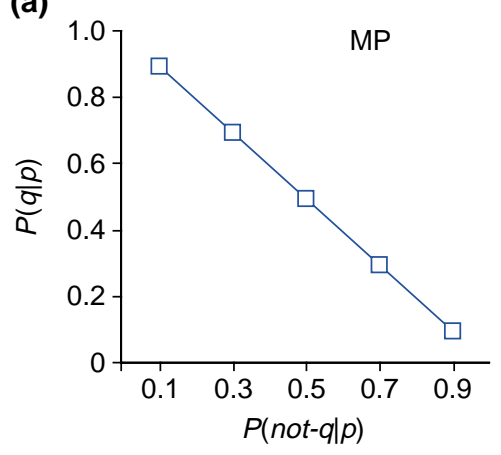

(c)

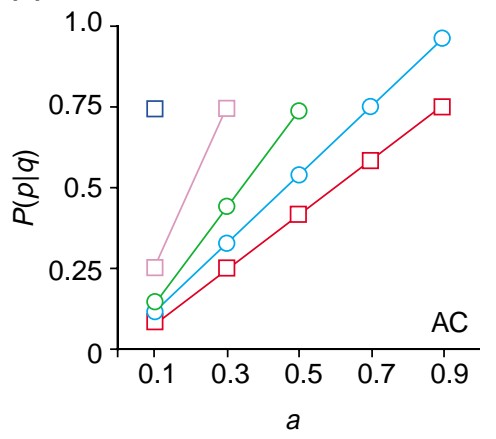

(b)

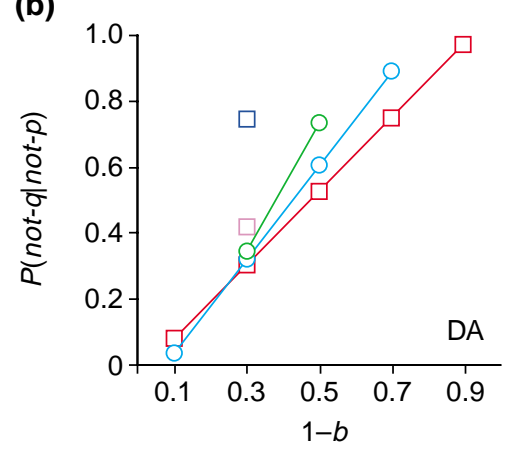

(d)

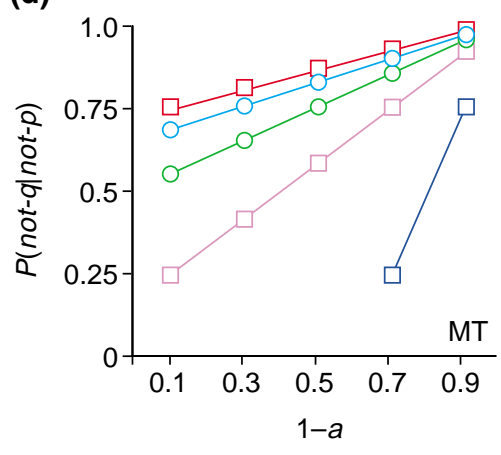

$\overline{T R E N D S}$ in Cognitive Sciences

Fig. I. How the probability that a conclusion should be drawn ( $y$-axis) varies as a function of the probability of the premise, $P$ (premise), and conclusion ( $x$-axis) for DA (b), for AC (c) and for MT (d). The probability that an MP inference should be drawn (a) relies only on the exceptions parameter $\varepsilon[P($ not- $q \mid p)]$. Where no value appears this is because it violates the assumptions of the probability model $(\varepsilon=0.25)$. Values of $P$ (premise) are: blue squares, 0.1 ; violet squares, 0.3 ; green squares, 0.5 ; blue circles, 0.7 ; red squares, 0.9 .

that conclusion because the car might not start if the petrol tank is empty. The petrol tank being empty provides an exception to the rule. These cases have been called 'additional antecedents', and it has been shown that they suppress the valid inferences modus ponens, MP, and modus tollens, MT (Ref. i). These effects are directly predicted by the probabilistic model, as can be seen from Fig. Ia. Additional antecedents correspond to high values of the exceptions parameter, $\varepsilon[P($ not $-q \mid p)]$. As Fig. la shows, as this parameter increases, the probability that the MP inference should be drawn decreases. This also happens for MT. More counter-intuitively, the model predicts that increases in this parameter should also decrease endorsements of the conclusions 'denying the antecedent' (DA) and 'affirming the consequent' (AC) (see main text, and Ref. j), and this effect has been observedk.

\section{References}

a Evans, J .St-B.T. (1977) Linguistic factors in reasoning. Q.J . Exp. Psychol. 29, 297-306

b Evans, J .St-B.T. et al. (1995) Bias in conditional inference: implications for mental models and mental logic. Q.J . Exp. Psychol . A 48, 644-670

c Evans, J .St-B.T. and Handley, S.J . (1999) The role of negation in conditional inference. Q.J .Exp. Psychol. 52, 739-770

d Oaksford, M. and Stenning, K. (1992) Reasoning with conditionals containing negated constituents. J . Exp. Psychol. Learn. Mem. Cognit. 18, 835-854

e Oaksford, M. and Chater, N . (1994)A rational analysis of theselection task as optimal data selection. Psychol. Rev. 101, 608-631

f Oaksford, M. etal. (2000) Probabilities and polarity biases in conditional inference. J . Exp. Psychol. Learn. Mem. Cognit. 26, 883-899

g Schroyens, W. et al. (2000) Conditional reasoning with negations: implicit and explicit affirmation or denial and the role of contrast classes. Think. Reason. 6, 221-251

h Schroyens, W. et al. (2000) Heuristicand analytic processes in conditional reasoning with negatives. J . Exp. Psychol. Learn. Mem. Cognit. 26, 1713-1734

i Byrne, R.M.J . (1989) Suppressing valid inferences with conditionals. Cognition 31, 1-21

j Oaksford, M. and Chater, N. Probabilities and pragmatics in conditional inference: suppression and order effects. In Reasoning and Decision Making (Hardman, D. and Macchi, L., eds), J ohn Wiley $\&$ Sons (in press)

k George, C. (1997) Reasoning from uncertain premises. Think. Reason. 3, 161-190 participants should now select not-q cards, regardless of rarity. According to the disinterested approach, however, card selection is independent of believability.
Wehave argued that the current evidence favours the disinterested approach with respect to the standard selection task ${ }^{50}$. H owever, there are task 


\section{Box 3. Explaining the selection task}

The behaviour of the 'information-gain model' in shown in Fig. I. In modelling performance on the selection task, perhaps the most important point to observe from these density plots is that when $P(p)$ and $P(q)$ are both small there is a region where the probability that the $q$ card should be selected is greater than the probability that the not-q card should be selected. When participants do the selection task, the most frequent response is to select the $p$ and the $q$ cards only. This behaviour is usually regarded as
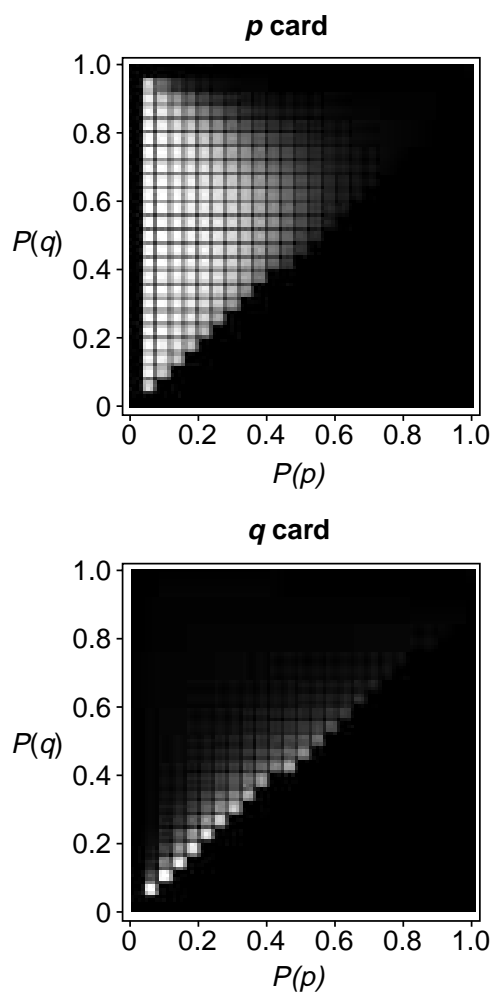

irrational. However, according to the information-gain model, if the probabilities of the antecedent and consequent are quite small ('the rarity assumption') then this selection of cards is the rational selection: these two cards are more informative about which hypothesis is true. That the probabilities of the antecedent and consequent should be low is consistent with the observation that the categories of natural language divide the world up quite finely. So, for example, very few things are tables,
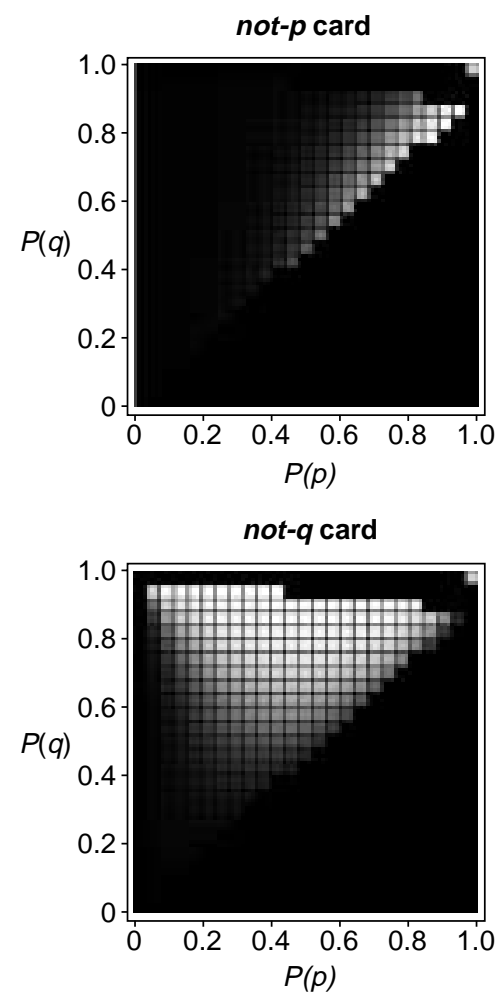

$\overline{T R E N D S}$ in Cognitive Sciences

Fig. I. The probabilities with which a card should be selected as a function of the probabilities of the antecedent $[P(p), x$-axes] and the consequent $[P(q), y$-axes] according to the revised information-gain model. The lighter the region the greater the probability that a card should be selected. These probabilities were calculated by transforming the information gains using a logistic selection-tendency function a The prior probabilities, $P\left(M_{t}\right)$ and $P\left(M_{D}\right)$ were set to 0.5 and the exceptions parameter $(\varepsilon)$ was set to 0.1 . Points in the lower-right triangular region in black violate the assumptions of the dependence model that $P(q)>P(p)(1-\varepsilon)$. cars or gorillas. There is now good evidence that rarity is the default assumption when people are testing ${ }^{\mathrm{b}, \mathrm{c}}$ or framing hypotheses ${ }^{d}$. The version of the model reported here meets a variety of theoretical objections, provides more rigorous fits to the data, and can account for more of the recent evidence on data selection.

In particular, the model provides detailed fits to the data obtained when negations are varied in the antecedents and consequents of the rules used in the selection task (see Box 2). For example, when this is done, participants select far more not- $q$ cards for the if $p$, then not- $q$ rule than the if $p$, then $q$ rule . Given the contrast set account of negation (see Box 2), Fig. I reveals why this should be the case. It is clear from the lower two panels in Fig. I that as the probability of the consequent, $P(q)$, increases, so the probability that the $q$ card should be chosen decreases but the probability that the not- $q$ card should be chosen increases. It has also been shown that card selections vary in this way when $P(p)$ and $P(q)$ are varied rather than negations ${ }^{f}$.

\section{References}

a Hattori, M. (1999) Theeffects of probabilistic information in Wason's selection task: an analysis of strategy based on the ODS model. Proc. 16th Annu. MtgJ pn. Cognit. Sci. Soc. 16, 623-626

b Anderson, J .R. and Sheu, C.F. (1995) Causa inferences as perceptual judgments. Mem. Cognit. 23, 510-524

c McKenzie, C.R.M. and Mikkelsen, L.A. (2000) The psychological side of Hempel's paradox of confirmation. Psychonomic Bull. Rev. 7, 360-366

d McKenzie, C.R.M. et al. Doconditional statements target rare events? Organiz. Behav. Hum. Decision Process. (in press)

e Evans, J .St-B.T. and Lynch, J .S. (1973) Matching bias in the selection task. Br. J . Psychol. 64, 391-397

f Oaksford, M. et al. (1999) Probabilistic effects in data selection. Think. Reason. 5, 193-243 manipulations ${ }^{57}$, particularly involving reasoning about practical action ${ }^{58}$, that require a decisiontheor etic approach. Indeed, in our original paper on the selection task $^{6}$ the decision-theoretic approach was taken to thesetask versions, and we still believe that this is appropriate. Nonetheless, whichever approach onetakes, despitesome contrary results ${ }^{17}$, it is now firmly establ ished that, '....no account of the selection task is sufficiently general if it cannot take account of theset size of $p$ and the set size of $q$ or the probability judgements which reflect these'15.

\section{Syllogistic reasoning}

The probabilistic approach has also been extended to syllogistic reasoning, in which a conclusion is drawn from two premises (e.g. 'Someof theartistsare beekepers'; 'All thebeekeepers arechemists'; therefore, 'Someof theartists arechemists'). In the probability heuristics model ${ }^{19}$ (PHM) the probabilistic interpretation of conditionals is extended to quantified claims: All, Some, None, and Some...not. In the contingency table of Fig. 1, if there are no exceptions, then the probability of the consequent 


\section{Box 4. Probabilistic heuristics for syllogistic reasoning}

There are three generation heuristics (G1-3) in the probability-heuristics model:

(G1) The min-heuristic: choose the quantifier of the conclusion to be the same as the quantifier in the least informative premise (the min-premise).

The most informative conclusions that can validly follow almost always follows this rule.

Some conclusions probabilistically-entail ('p-entail') other conclusions. For example, if $A$ Il $X$ are $Y$, then it is probable that Some $X$ are $Y$ (this will follow as long as there are some $X \mathrm{~s}$ ). Thus, the second heuristic is:

(G2) p-entailments: the next most preferred conclusion will be the $p$-entailment of the conclusion predicted by the minheuristic (the 'min-conclusion').

Heuristics G1 and G2 specify the quantifier of the conclusion. The third heuristic, the attachment-heuristic, specifies the order of end terms in the conclusion:

(G3) Attachment-heuristic: if just one of the possible conclusion subject noun phrases matches the subject noun phrase of just one premise, then the conclusion has that subject noun phrase.* (where subject noun is: $A / l X, A / l Z$, Some $X$, Some $Z$, etc.)

Crucially, Some $X$ are not Zand Some $X$ are Zhave the same subject noun phrase: 'Some $X$ '. We illustrate these heuristics with an example (where '_' stands as a place holder for a subject of predicate term in the mental representation of the putative conclusion):

$\begin{array}{ll}\text { SomeZareY } & \text { (min-premise) } \\ \text { All Y areX } & \text { (max-premise) } \\ \text { Some_are_- } & \text { (by min) } \\ \text { SomeZZareX } & \text { (by attachment) } \\ \text { Some_are not_- } & \text { (by p-entailment) } \\ \text { SomeZZare notX } & \text { (by attachment) }\end{array}$

By the min-heuristic, the conclusion is Some. The min-premise has an end term (Z) as its subject. Therefore, by attachment, the conclusion will have $Z$ as its subject term, and the form Some $Z$ are $X$. The $p$-entailment of the min-conclusion is Some $Z$ are not $X$ which is predicted to be the next most endorsed conclusion.

G1-G3 generate syllogistic conclusions. It is assumed that people are rarely able to test these conclusions for $p$-validity (or, for that matter, logical validity). However, it is argued that people use two test heuristics that provide a fast and frugal estimate of how likely the conclusion generated by G1-G3 is to be informative and $p$-valida:

(T1) The max-heuristic: be confident in the conclusion generated by G1-G3 in proportion to the informativeness of the most informative premise (the max-premise).

(T2) The Some...not-heuristic: avoid producing or accepting Some...not conclusions, because they are so uninformative relative to other conclusions.

*Wethank Geoff Goodwin (BrisbaneUniversity, Australia; pers. commun.) for pointing out an error in theoriginal formulation of G3 (Ref. a). Although theexampleis consistent with the definition we providehere, theoriginal definition was toonarrow.

\section{Reference}

a Chater, N. and Oaksford, M. (1999) The probability heuristics model of syllogistic reasoning. Cognit. Psychol. 38, 191-258 given theantecedent, $(P[q \mid p])$, is 1 . The conditional and the universal quantifier All have the same underlyinglogical form: $\forall \mathrm{x}(\mathrm{P}(\mathrm{x}) \Rightarrow \mathrm{Q}(\mathrm{x}))$.

Consequently universal daims, such as All Ps areQs, were interpreted as asserting that the probability of the predicate term $(Q)$ given the subject term $(P)$ is 1; that is, $P(Q \mid P)=1$. Probabilisticmeanings for the other quantifiers are then easily defined: None, $P(Q \mid P)=0$; Some, $P(Q \mid P)>0$; Some...not, $P(Q \mid P)<1$.

Given these probabilistic interpretations it is possibleto prove which condusions follow, probabilistically, for all 64 possible syllogisms (i.e. which syllogisms are 'p-valid'). M oreover, given theseinterpretations, and again making the rarity assumption (seeabove on the selection task), the quantifiers can beordered in terms of how informative they are: All > Some > N one > Some...not. It turns out that a simpleset of heuristics defined with respect totheinformativeness of the premises can successfully predict the p-valid conclusion if there is one (see Box 4).

Themost important is themin-heuristic, which states that thecondusion will havetheform of the least informative premise. Sofor example, a p-valid syllogism, such as AII B areA; SomeB arenot C, yields the condusion SomeA arenot $C$. This simple heuristic captures the form of the conclusion for most p-valid syllogisms (see Box 5). Moreover, if overgeneralized to the invalid syllogisms, the conclusions it suggests match the empirical data very well. Other heuristics determinetheconfidence that people have in their conclusions and theorder of terms in the conclusion (see Box 4).

The most important feature of PHM is that it can general ize to syllogisms containing quantifiers, such as Most and $\mathrm{F}$ ew, that have no logical interpretation. In terms of Fig. 1 these quantifiers are used when thereare some (M ost) or many (F ew) exceptions. So themeaning of Most is: $1-\Delta<\mathrm{P}(\mathrm{Q} \mid \mathrm{P})<1$, and the meaning of $\mathrm{F}$ ew is: $0<\mathrm{P}(\mathrm{Q} \mid \mathrm{P})<\Delta$, where $\Delta$ is small. These interpretations lead to the following order of informativeness: All $>$ Most $>$ F ew $>$ Some $>$ N one $>$ Some...not. Consequently, PHM uniquely makes predictions for the 144 syllogisms that are produced when M ost and Few are combined with the standard logical quantifiers. Weshowed previously that these heuristics pick out the p-valid condusions for these new syllogisms, and reported experiments that confirm the predictions of PHM when Most and Few are used in syllogistic arguments ${ }^{18}$.

There has already been some further work on syllogistic reasoning consistent with PHM (Refs 19,20). For example, themin-heuristic captures the novel distinction between strong and weak possible conclusions $^{19}$. Take the syllogism All Y areX; SomeZ areY. The conclusion SomeZ areX, follows necessarily from these premises; NoZ areX, is impossible, but SomeZ arenot X and All Z areX are possible. Some possiblecond usions are endorsed as strongly as necessary conclusions (e.g. SomeZ are 


\section{Box 5. Explaining syllogistic reasoning}

Table I shows the results of a meta-analysis showing the weighted average of correct responses to the logically valid syllogisms ${ }^{a-d}$. The pairs, for example, $(X, Z)$, in the syllogistic premises are ordered to show their status as the subject (first) or the predicate (second) term. The logically valid conclusion and the number of mental models required to reach that conclusion are also shown.

These data reveal how the heuristics of PHM can explain the data. Note that all the syllogisms above the red line are drawn more often than all those below it (with one exception). Moreover, all the syllogisms above the blue line are drawn more often than all of those below it. All the syllogisms above the red line conform perfectly to the min-heuristic ( $\mathrm{G} 1$; see Box 4); those below, although not violating the min-heuristic (the conclusion is less informative than the minconclusion), do not conform to it. All those syllogisms that are below the blue line only have a very uninformative Some...not conclusion (T2) and interestingly this conclusion is drawn only as often as Nonewhich is the min-conclusion. Moreover, Some...notis a p-entailment of None (G2). Finally, all those syllogisms above the red line also have very informative max-premises (T1). Thus, it would seem that a set of very simple heuristics defined with respect to the informativeness of the premises can explain the differences in performance for the logically valid syllogisms.

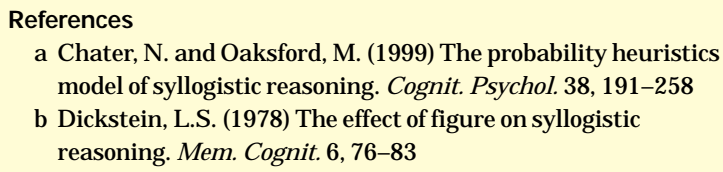

not X) and some are endorsed as weakly as impossible conclusions (e.g. All Z areX). Possible strong condusions all conform to themin-heuristic; that is, they either match themin-premiseor areless informativethan the min-premise. Possibleweak condusions all violatethemin-heuristic (bar one); that is, they have conclusions that aremore informativethan themin-premise.

At first sight, mental-models theory al so explains this result. Strong possible conclusions are those licensed in the initial model but not in subsequent models. Weak possibleconclusions are thoselicensed only in non-initial models. This means that the conclusions recommended by themin-heuristic correspond tothoselicensed by initial mental models. This is an interesting coincidence, as neither theory was constructed with the distinction between strong and weak possible conclusions in mind. However, it is unclear why mental-models theory licenses theseinitial models. The only suggestion that has been made is that it is an 'emergent
Table I. Meta-analysis of syllogisms

\begin{tabular}{|c|c|c|c|}
\hline Syllogism & Conclusion & $\begin{array}{l}\text { No. of } \\
\text { mental } \\
\text { models }\end{array}$ & Mean ${ }^{a}$ \\
\hline$A / /(Y, X), A / /(Z, Y)$ & All & 1 & 89.87 \\
\hline$A / /(\mathrm{X}, \mathrm{Y}), A / /(\mathrm{Y}, \mathrm{Z})$ & All & 1 & 75.32 \\
\hline$A / /(Y, X)$, Some $(Z, Y)$ & Some & 1 & 86.71 \\
\hline Some(Y,X), Al/(Y,Z) & Some & 1 & 87.97 \\
\hline$A / /(\mathrm{Y}, \mathrm{X})$, Some $(\mathrm{Y}, \mathrm{Z})$ & Some & 1 & 88.61 \\
\hline Some(X,Y), Al/(Y,Z) & Some & 1 & 86.71 \\
\hline$N o(Y, X), A / /(Z, Y)$ & No & 1 & 92.41 \\
\hline$A / /(X, Y), N o(Z, Y)$ & No & 1 & 84.81 \\
\hline$N o(X, Y), A / /(Z, Y)$ & No & 1 & 88.61 \\
\hline$A / /(\mathrm{X}, \mathrm{Y}), N o(\mathrm{Y}, \mathrm{Z})$ & No & 1 & 91.14 \\
\hline All $(\mathrm{X}, \mathrm{Y})$, Some...not(Z,Y) & Some...not & 2 & 67.09 \\
\hline Some...not $(X, Y), A / /(Z, Y)$ & Some...not & 2 & 56.33 \\
\hline Al/(Y,X), Some...not $(\mathrm{Y}, \mathrm{Z})$ & Some...not & 2 & 66.46 \\
\hline Some...not(Y,X), Al/(Y,Z) & Some...not & 2 & 68.99 \\
\hline Some(Y,X), No(Z,Y) & Some...not & 3 & 66.46 \\
\hline$N o(Y, X)$, Some $(\mathrm{Z}, \mathrm{Y})$ & Some...not & 3 & 16.46 \\
\hline Some $(X, Y), N o(Z, Y)$ & Some...not & 3 & 51.90 \\
\hline$N o(X, Y)$, Some $(Z, Y)$ & Some...not & 3 & 30.38 \\
\hline Some $(\mathrm{Y}, \mathrm{X}), \mathrm{No}(\mathrm{Y}, \mathrm{Z})$ & Some...not & 3 & 48.10 \\
\hline No(Y,X), Some $(\mathrm{Y}, \mathrm{Z})$ & Some...not & 3 & 32.91 \\
\hline Some $(\mathrm{X}, \mathrm{Y}), \operatorname{No}(\mathrm{Y}, \mathrm{Z})$ & Some...not & 3 & 26.58 \\
\hline No(X,Y), Some $(\mathrm{Y}, \mathrm{Z})$ & Some...not & 3 & 44.30 \\
\hline
\end{tabular}

C J ohnson-Laird, P.N. and Bara, B.G. (1984) Syllogistic inference. Cognition 16, 1-62

d J ohnson-Laird, P.N. and Steedman, M. (1978) The psychology of syllogisms. Cognit. Psychol. 10, 64-99 property' of the computer program that embodies the mental models theory. But no account of the principles underlying initial model construction that can explain why this property emerges has been forthcoming. In summary, PHM would appear to be gaining some empi rical support.

\section{Everyday and logical inference revisited}

Finally, we consider a potential problem for the probabilistic approach. Some people do reason logically some of the time 37,38 and they tend to score more highly on IQ tests ${ }^{38}$. This observation has been interpreted as supporting a dual-process view ${ }^{10}$. 'System-1' processes are automatic, unconscious and based on implicitly acquired world knowledge. 'System-2' processes are control led, analytical and based on explicitly acquired formal rules. The probabilistic approach provides a computationallevel theory of System-1 processes in which the probabilities involved are consi dered as summary statistics computed over world knowledge. On this 


\section{Questions for future research}

- The probabilistic approach has largely concentrated on the computational level of explanation. A challenge is to move to the algorithmic level and to propose theories of the actual mechanisms that implement these processes in the mind/brain. In this regard Bayesian networks ${ }^{47,48}$ look promising.

- We regard the probabilities in the models as deriving from world knowledge. But providing an account of the storage and retrieval of world knowledge on the scale required to explain human cognition and reasoning is not a tractable problem ${ }^{60}$. Major breakthroughs in the computer science of knowledge-based systems will probably be required before complete explanations of human reasoning will be possible.

- Other accounts of human reasoning, in particular mental models, have been extended beyond the coretasks discussed in this paper. The viability of the probabilistic approach depends on showing that it can be extended to these areas and continue to make novel predictions on the way.

- How human reasoning relates to information processing in the brain has not been seriously addressed by any theory (even though studies of the relationship between reasoning and the brain have begun ${ }^{61,62}$ ). The probabilistic approach might be helpful here because of the relationship between Bayesian networks and neural networks ${ }^{63}$. That is, probability theory could provide the right language in which to connect 'higher' level cognition to brain-style computation. view, most reasoning involves only System-1 processes. However, some people, especially the more intelligent, might acquire explicit logical rules, either culturally or by explicit tuition. This is consistent with the probabilistic approach where the possibility has already been raised that some people might use System-2 processes to test conclusions generated by System-1 (Ref. 18).

The critical question is the balance of System-1 versus System- 2 processes in human reasoning. Most recent theorizing has been about System-2 processes ${ }^{30,31}$. However, results from the selection task ${ }^{59}$ suggest that, at most, $10 \%$ of university students arecapable of engaging System-2 processes when reasoning. If, as this result suggests, most reasoning invokes only System-1 processes, then surely this is where reasoning researchers should be looking. A probabilistic approach tothese processes explains people's performance in thelaboratory as a rational attempt to makesense of thetasks they are set, by applying strategies adapted for coping with the uncertainty of theeveryday world. It is these strategies that create the appearance of biased and irrational reasoning when compared with the standard provided by formal logic.

\section{References}

1 Evans, J .St-B.T. et al. (1993) Human Reasoning, Erlbaum

2 Manktelow, K. (1999) Reasoning and Thinking, Psychology Press

3 Stein, E. (1996) Without Good Reason, Oxford University Press

4 Stich, S. (1985) Could man bean irrational animal? Synthese64, 115-135

5 Wason, P.C. (1968) Reasoning about a rule. Q.J .Exp. Psychol. 20, 273-281

6 Oaksford, M. and Chater, N. (1994)A rational analysis of the selection task as optimal data selection. Psychol. Rev. 101, 608-631

7 Oaksford, M. and Chater, N. (1998) A revised rational analysis of the selection task: exceptions and sequential sampling. In Rational Models of Cognition (Oaksford, M. and Chater, N., eds), pp. 372-398, Oxford University Press

8 Hattori, M. (1999) The effects of probabilistic information in Wason's selection task: an analysis of strategy based on the ODS model. Proc. 16th Annu. MeetingJ pn. Cognit. Sci. Soc. 16, 623-626

9 Klauer, K.C. (1999) On thenormativejustification for information gain in Wason's selection task. Psychol. Rev. 106, 215-222

10 Evans, J St-B.T. and Over, D.E. (1996) Rationality in the selection task: epistemic utility versus uncertainty reduction. Psychol. Rev. 103, 356-363

11 Nickerson, R.S. (1996) Hempel's paradox and Wason's selection task: logical and psychological puzzles of confirmation. Think. Reason. 2, 1-32

12 Oaksford, M., et al. (1999) Probabilistic effects in data selection. Think. Reason. 5, 193-243

13 Oaksford, M. et al. (1997) Optimal data selection in the reduced array selection task (RAST). J . Exp. Psychol . Learn. Mem. Cognit. 23, 441-458

14 Green. D.W. and Over, D.E. (1997) Causal inference, contingency tables and the selection task. Curr. Psychol. Cognit. 16, 459-487
15 Green, D.W. and Over, D.E. (2000) Decision theoretical effects in testing a causal conditional. Curr. Psychol. Cognit. 19, 51-68

16 Manktelow, K.I. etal. (1995) Probabilisticfactors in deontic reasoning. Think. Reason. 1, 201-220

17 Oberauer, K. et al. (1999) Bayesian rationality for the Wason selection task? A test of optimal data selection theory. Think. Reason. 5, 115-144

18 Chater, N. and Oaksford, M. (1999) The probability heuristics model of syllogistic reasoning. Cognit. Psychol. 38, 191-258

19 Evans, J .St-B.T. et al. (1999) Reasoning about necessity and possibility: a test of themental model theory of deduction.J . Exp. Psychol. Learn. Mem. Cognit. 25, 1495-1513

20 Newstead, S.E. etal. (1999). Falsifying mental models: testing the predictions of theories of syllogistic reasoning. Mem. Cognit. 27, 344-354

21 Oaksford, M. et al. (2000) Probabilities and polarity biases in conditional inference. J . Exp. Psychol. Learn. Mem. Cognit. 26, 883-899

22 Oaksford, M. and Chater, N. Probabilities and pragmatics in conditional inference: suppression and order effects. In Reasoning and Decision Making (Hardman, D. and Macchi, L., eds), $J$ ohn Wiley \& Sons (in press)

23 Schroyens, W. et al. (2000) Conditional reasoning with negations: implicit and explicit affirmation or denial and the role of contrast classes. Think. Reason. 6, 221-251

24 Schroyens, W. etal. (2000) Heuristic and analytic processes in conditional reasoning with negatives. J . Exp. Psychol. Learn. Mem. Cognit. 26, 1713-1734

25 Chan, D. and Chua, F. (1994) Suppression of valid inferences: syntactic views, mental models, and relativesalience. Cognition 53, 217-238

26 George, C. (1997) Reasoning from uncertain premises. Think. Reason. 3, 161-190

27 Liu, I. etal. (1996) A probabilisticinterpretation of 'If-then'. Q.J . Exp. Psychol. A 49, 828-844
28 Stevenson, R.J . and Over, D.E. (1995) Deduction from uncertain premises. Q.J . Exp. Psychol. A $48,613-643$

29 George, C. (1999) Evaluation of the plausibility of a conclusion from several arguments with uncertain premises. Think. Reason. 5, 245-281

30 Rips, L.J . (1994) ThePsychol ogy of Proof, MIT Press

31 J ohnson-Laird, P.N. and Byrne, R.M.J . (1991) Deduction, Erlbaum

32 Evans, J .St-B. T. and Over, D. (1996) Rationality and Reasoning, Psychology Press

33 Chater, N. and Oaksford, M. (1999) Ten years of therational analysis of cognition. Trends Cognit. Sci. 3, 57-65

34 Anderson, J .R. (1990) TheAdaptiveCharacter of Thought, Erlbaum

35 Anderson, J .R. (1991) I s human cognition adaptive? Behav. Brain Sci. 14, 471-517

36 Oaksford, M. and Chater, N., eds (1998) Rational Models of Cognition, Oxford University Press

37 Braine, M.D.S. and O'Brien, D.P. (1998) Mental Logic, Erlbaum

38 Stanovich, K.E. and West, R.F. (2000) Individual differences in reasoning: implications for therationality debate. Behav. Brain Sci. 23, 645-726

39 Oaksford, M. and Chater, N. (1998) Rationality in an Uncertain World, Psychology Press

40 Oaksford, M. and Chater, N. (1995) Theories of reasoning and the computational explanation of everyday inference. Think. Reason. 1, 121-152

41 Oaksford, M. and Chater, N. (1991) Against logicist cognitivescience. Mind Lang. 6, 1-38

42 Oaksford, M. and Chater, N. (1992) Bounded rational ity in taking risks and drawing inferences. Theory Psychol. 2, 225-230

43 Oaksford, M. and Chater, N. (1993) Reasoning theories and bounded rationality. In Rationality (Manktelow, K.I . and Over, D.E., eds), pp. 31-60, Routledge 
44 Chater, N. and Oaksford, M. (1993) Logicism, mental models and everyday reasoning. Mind Lang. 8, 72-89

45 Adams, E. (1966) Probability and the logic of conditionals. In Aspects of I nductive Logic (Hintikka, J . and Suppes, P., eds), pp. 265-316, North Holland

46 Adams, E. (1975) The Logic of Conditionals: An Application of ProbabilityTheory to Deductive Logic, Reidel

47 Pearl, J . (1988) Probabilistic Reasoning in Intelligent Systems, Morgan Kaufmann

48 Pearl, J . (2000) Causality, CambridgeUniversity Press

49 Shafer, G. (1976) A Mathematical Theory of Evidence, Princeton University Press

50 Chater, N. and Oaksford, M. (1999) I nformation gain vs. decision-theoretic approaches to data selection. Responseto Klauer. Psychol. Rev. $106,223-227$
51 Oaksford, M. and Chater, N. (1996) Rational explanation of the sel ection task. Psychol. Rev. 103, 381-391

52 Laming, D. (1996) On theanalysis of irrational data sel ection: a critique of Oaksford and Chater (1994). Psychol. Rev. 103, 364-373

53 Almor, A. and Sloman, S.A. (1996) Is deontic reasoning special? Psychol. Rev. 103, 374-380

54 Green, D.W. (2000) Review of Rationalityin a Uncertain World: Essays on the CognitiveScience of Human Reasoning. Q.J . Exp. Psychol A 53, 281-283

55 Green, D.W. and Over, D.E. (1998) Reaching a decision. A reply to Oaksford. Think. Reason. 4, 231-248

56 Evans, J .St-B.T. (1999) Rational analysis of illogical reasoning (Review of Rationality in an Uncertain World: Essays on theCognitiveScience of Human Reasoning). Contemp. Psychol . 44, 461-463
57 Sperber, D. et al. (1995) Relevancetheory explains theselection task. Cognition 57, 31-95

58 Manktelow, K.I. and Over, D.E. (1993) Rationality: Psychological and Philosophical Perspectives, Routledge

59 Stanovich, K.E. and West, R.F. (1998) Cognitive ability and variation in selection task performance. Think. Reason. 4, 193-230

60 Pylyshyn, Z.W. and Ford, K.M. (1995) TheRobot's Dilemma Revisited, Ablex Publishing

61 Wharton, C.M. and Grafman, J . (1998) Deductive reasoning and the brain. Trends Cognit. Sci. 2, 54-59

62 Houde, O. et al. (2000) Shifting from the perceptual brain tothelogical brain: theneural impact of cognitiveinhibition training. J . Cogn. Neurosci. 12, 721-728

63 McClelland, J .L. (1998) Connectionist models and Bayesian inference. In Rational Models of Cognition (Oaksford, M. and Chater, N., eds), pp. 21-53, Oxford University Press

\section{Shedding light on brain function: the event-related optical signal}

\section{Gabriele Gratton and Monica Fabiani}

\section{One of the basic goals of cognitive psychology is the analysis of the covert processes that occur between stimulus and response. In the past 20-30 years, the tools available to cognitive psychologists have been augmented by a number of imaging techniques for studying the 'brain in action' in a non-invasive manner. These techniques have their strength in either temporal or spatial information, but not both. We review here recent advances of a new approach, the event-related optical signal (EROS). This method allows measurements of the time course of neural activity in specific cortical structures, thus combining good spatial and temporal specificity. As an example, we show how EROS can be used to distinguish between serial and parallel models of information processing.}

A new brain imaging technique, theevent-related optical signal (EROS), combines spatial resolution of better than a centimetrewith temporal resolution of the order of milliseconds, which makes it ideal to study thetime course of neural activity in localized cortical areas. Theimaging signal reflects changes in the optical scattering properties of brain tissuethat are concurrent with neuronal activity. Because the same instrument that measures EROS can al so detect hemodynamic phenomena that occur subsequent to neuronal activity, this techniqueis uniquely suited tostudy therelationship between neuronal and

Gabriele Gratton* Monica Fabiani Beckman Institute, University of Illinois, 405 N. Matthews Ave, Urbana, IL 61801, USA. *e-mail:

GrattonG@uinc.edu vascular effects (neurovascular coupling). Presently, limitations of thetechniqueinclude reduced penetration (sothat only structures within $3-5 \mathrm{~cm}$ from the surface of the head can bestudied) and relatively low signal-to-noise ratio, which in reality requires that data is averaged across subjects.
Two major classes of functional brain imaging methods are most used at present: hemodynamic techniques (such as PET and FMRI), which are particularly useful for visualizing whereneural activity occurs; and electrophysiol ogical techniques (such as event-related brain potentials- ERPs, and magnetoencephalography-MEG), which are particularly useful for determining when activity occurs ${ }^{1}$. Both classes of techniquehave provided important data for understanding the brain processes underlying cognitivefunction ${ }^{2-4}$. However, each of them special izes in a particular domain, but provides limited information in the other domain. For this reason, several investigators have proposed that they becombined to provide spatio-temporal maps of brain activity5,6. This approach requires the assumptions that the different signals are co-localized and respond si milarly to experimental manipulation, assumptions that need to be val idated in each case.

Light scattering changes associated with neural activity EROS is based on a different approach from previous methods, and in particular on thefact that lightscattering properties of neural tissuechangewhen the tissue is active. This phenomenon was first demonstrated more than 50 years $\mathrm{ago}^{7}$, and has been used to study the behavior of largenumbers of individual neurons in parallel ${ }^{8}$. Rector et al. have shown that thesamemethod can beused tostudy 